\title{
Prevalence and Appropriateness of Indwelling Urinary Catheters in Japanese Hospital Wards: A Multicenter Point Prevalence Study
}

\author{
Kohta Katayama ( $\sim$ pine77717@gmail.com ) \\ International University of Health and Welfare Narita Hospital \\ Jennifer Meddings \\ VA Ann Arbor Healthcare System \\ Sanjay Saint \\ VA Ann Arbor Healthcare System \\ Karen Fowler \\ VA Ann Arbor Healthcare System \\ David Ratz \\ VA Ann Arbor Healthcare System \\ Yasuaki Tagashira \\ Tokyo Metropolitan Tama Medical Center \\ Yumi Kawamura \\ Mimihara General Hospital \\ Tatsuya Fujikawa \\ Mitoyo General Hospital \\ Sho Nishiguchi \\ Shonan Kamakura General Hospital \\ Naomi kayauchi \\ Mito Kyodo General Hospital \\ Nobumasa Takagaki \\ Nobumasa Clinic \\ Yasuharu Tokuda \\ Muribushi Okinawa Center for Teaching Hospitals \\ Akira Kuriyama \\ Kurashiki Central Hospital
}

\section{Research Article}

Keywords: Urinary Catheters, Infection Control, Prevalence, Patient Safety, Japan

Posted Date: November 2nd, 2021

DOI: https://doi.org/10.21203/rs.3.rs-970770/v1 
License: (c) (7) This work is licensed under a Creative Commons Attribution 4.0 International License. Read Full License

Version of Record: A version of this preprint was published at BMC Infectious Diseases on February 21st, 2022. See the published version at https://doi.org/10.1186/s12879-022-07162-3. 


\section{Abstract}

Background: Indwelling urinary catheters are commonly used in hospitalized patients, which can lead to the development of urinary catheter complications, including catheter-associated urinary tract infection (CAUTI). Limited reports on the appropriateness of urinary catheter use exist in Japan. This study investigated the prevalence and appropriateness of indwelling urinary catheters, and the incidence of CAUTI in non-intensive care unit (non-ICU) wards in Japanese hospitals.

Methods: This prospective observational study was conducted in 7 non-ICU wards from 6 hospitals in Japan from October 2017 to June 2018. At each hospital the study teams evaluated urinary catheter prevalence through inperson bedside evaluation for at least 5 days of each week for 3 months. Catheter associated urinary tract infection (CAUTI) incidence and appropriateness of catheter use was collected via chart review.

Results: We assessed 710 catheter-days over 5528 patient-days. The mean prevalence of indwelling urinary catheter use in participating wards was 13\% (range: $5 \%$ to 19\%), while the mean incidence of CAUTI was 9.86 per 1000 catheter-days (range: 0 to 33.90 ). Approximately $66 \%$ of the urinary catheter days assessed had an appropriate indication for use (range: $17 \%$ to $81 \%$ ). A physician's order for catheter placement was present in only $10 \%$ of catheterized patients.

Conclusion: This national prospective study provides epidemiological information about the appropriate use of urinary catheters in Japanese non-ICU wards. A multimodal intervention may help improve the appropriate use of urinary catheters.

\section{Introduction}

Indwelling urinary catheters are commonly used in hospitalized adults, including approximately $20 \%$ of hospitalized patients in Western countries. ${ }^{1}$ Urinary catheter complications are also common, including catheter-associated urinary tract infection (CAUTI) with a reported prevalence of $6 \%$ of hospital-associated infections (HAIs) in the United States $^{2}$ as well as causing patient discomfort from urethral trauma, immobility, and inadvertent removal. ${ }^{3}$ The most effective intervention to prevent both infectious and non-infectious harms is avoiding inappropriate indwelling urinary catheter use. ${ }^{4,5}$

Limited reports on the appropriateness of urinary catheter use exist in Asia. ${ }^{6}$ One study from Japanese intensive care units (ICUs) reported that the point prevalence of urinary catheters was $76 \%$ with only $54 \%$ of those catheters considered appropriate. ${ }^{7}$ Urinary catheter use in Japanese non-ICU wards has not been previously studied.

We thus sought to estimate the prevalence and appropriateness of indwelling urinary catheters, and to determine the incidence of CAUTI in non-critical care units in Japanese hospitals.

\section{Methods}

This prospective study was conducted at multiple hospitals in Japan from October 2017 to June 2018. We enrolled 7 wards from 6 community hospitals. The participating hospitals included one university-affiliated community hospital (Hospital A), and a variety of public (Hospitals B \& C) and private (Hospitals D, E, \& F) hospitals. Each participating unit had between 40 and 60 adult beds. Additional participating hospital and unit characteristics can be found in the 
Supplementary Appendix. Only Hospital B employed physicians specializing in infectious diseases. The ethics committee of each participating hospital approved the study protocol.

The study teams at each hospital evaluated urinary catheter prevalence through in-person bedside evaluation, appropriateness of catheter use via chart review, and CAUTI incidence at least 5 days of each week for 3 months. Study observers - either physicians or research nurses - used the 2009 Healthcare Infection Control Practice Advisory Committee (HICPAC) guidelines and the Ann Arbor Criteria for Appropriate Urinary Catheter Use to determine catheter appropriateness. ${ }^{6,9}$ Indwelling urinary catheters in critically ill patients were deemed appropriate when medical staff required hourly urine volume measurement. Even in non-ICU wards, appropriate urine output monitoring by an indwelling urinary catheter was sometimes felt to be required to manage patients with electrolyte abnormalities or decompensated heart failure. Urinary catheters in patients who required prolonged strict immobilization for therapeutic purposes, such as pelvic fracture and unstable thoracic or lumbar spine, were also considered appropriate. The beside nurses were asked for their assessment of catheter indication. Study observers in all hospitals, except for Hospital C, also independently assessed the indication for every urinary catheter through medical record review.. The medical records were reviewed for orders for urinary catheter placement as well as documentation of urinary catheter presence.

\section{STATISTICAL ANALYSES}

The primary outcomes were: 1) proportion of patients in non-ICU wards with an indwelling urinary catheter; 2) proportion of patients with a urinary catheter that had an appropriate indication based on independent assessment; and 3) CAUTI incidence. We also assessed how often the urinary catheter's use was documented in the medical record, and if there was a physician order for placement of the urinary catheter. Data analysis was conducted through SAS software, version 9.4 (Cary, North Carolina).

\section{Results}

Data were assessed for 710 catheter-days over 5528 patient-days. The prevalence of urinary catheters was $13 \%$ (range: $5-19 \%$ ). Table 1 lists urinary catheter utilization by hospital. One hospital (Hospital C) did not conduct an independent review of urinary catheter indication and therefore their data on catheter appropriateness were excluded. Based on the observers' independent assessment, urinary catheters were deemed appropriate in 371 of the 586 catheter-days (63\%; range: 17-81\%). The total incidence of CAUTI was 9.86 per 1000 catheter-days. 
Table 1

Baseline Urinary Catheter Point Prevalence in Japanese Hospitals

\begin{tabular}{|c|c|c|c|c|c|c|c|c|}
\hline Hospital & $\begin{array}{l}\text { Patient } \\
\text { Days }\end{array}$ & $\begin{array}{l}\text { Foley } \\
\text { Days }\end{array}$ & $\begin{array}{l}\text { Point } \\
\text { Prevalence }\end{array}$ & $\begin{array}{l}\text { Documented } \\
\text { in Record }\end{array}$ & $\begin{array}{l}\text { Order for } \\
\text { Placement }\end{array}$ & $\begin{array}{l}\text { Appropriate } \\
\text { Indications } \\
\text { per Bedside } \\
\text { Nurse } \\
\text { Assessment }\end{array}$ & $\begin{array}{l}\text { Appropriate } \\
\text { Indications } \\
\text { per } \\
\text { Independent } \\
\text { Observer } \\
\text { Assessment }\end{array}$ & CAUTI \\
\hline $\begin{array}{l}\text { Hospital } \\
\text { A }\end{array}$ & 826 & 41 & $5 \%$ & $8(20 \%)$ & $1(2 \%)$ & $17(41 \%)$ & $7(17 \%)$ & 0 \\
\hline $\begin{array}{l}\text { Hospital } \\
\text { B }\end{array}$ & 717 & 134 & $19 \%$ & 134 (100\%) & $0(0 \%)$ & $100(75 \%)$ & 78 (58\%) & 0 \\
\hline $\begin{array}{l}\text { Hospital } \\
\text { C - Unit } \\
1\end{array}$ & 564 & 54 & $10 \%$ & $51(94 \%)$ & $0(0 \%)$ & $50(93 \%)$ & $N / A^{*}$ & 0 \\
\hline $\begin{array}{l}\text { Hospital } \\
\text { C - Unit } \\
2\end{array}$ & 588 & 70 & $12 \%$ & $70(100 \%)$ & $0(0 \%)$ & 49 (70\%) & $N / A^{*}$ & 1 \\
\hline $\begin{array}{l}\text { Hospital } \\
\text { D }\end{array}$ & 925 & 128 & $14 \%$ & $119(93 \%)$ & $8(6 \%)$ & $101(79 \%)$ & $89(70 \%)$ & 2 \\
\hline $\begin{array}{l}\text { Hospital } \\
\text { E }\end{array}$ & 893 & 118 & $13 \%$ & 115 (97\%) & $35(30 \%)$ & 65 (55\%) & $63(53 \%)$ & 4 \\
\hline $\begin{array}{l}\text { Hospital } \\
\text { F }\end{array}$ & 1015 & 165 & $16 \%$ & $152(92 \%)$ & $26(16 \%)$ & $136(82 \%)$ & $134(81 \%)$ & 0 \\
\hline Total & 5,528 & 710 & $13 \%$ & 649 (91\%) & 70 (10\%) & $518(73 \%)$ & $371(63 \%)$ & 7 \\
\hline
\end{tabular}

The most common indication for urinary catheter use was acute urinary retention or bladder outlet obstruction (as assessed by bedside nurses (38\%) and observers (35\%)), followed by need for accurate measurement of input and output in critically ill patients (19\% bedside nurses, $18 \%$ observers). Common indications for urinary catheters that were deemed inappropriate included monitoring input and output in non-critically ill patients (15\% bedside nurses, $12 \%$ observers) and no apparent reason for catheter use ( $2 \%$ nurses, $12 \%$ observers). Although urinary catheters were documented in the medical record in $91 \%$ of patients, a physician's order for catheter placement was present in only $10 \%$ of records for catheterized patients.

\section{Discussion}

Our multicenter study found that urinary catheters were present in $13 \%$ of patients, with only $63 \%$ of assessed catheter-days meeting appropriate indications for use. The prevalence of urinary catheters in this study was similar to that in the US. ${ }^{1,2}$ Urinary catheter prevalence in our study was much lower than that reported in Japanese ICUs $(76 \%){ }^{8}$ likely due to lower acuity of illness.

The proportion of inappropriate urinary catheter use and CAUTI incidence in our study (9.86 infections per 1000 catheter-days) was higher than reports from the US (1.54-2.28 infections per 1000 catheter-days). ${ }^{1}$ Similar to prior 
studies, ${ }^{7}$ many of these inappropriate catheters either had no apparent reason for use, or were used for urine output management in non-critically ill patients. Interventions such as catheter reminders and stop orders have been reported to be effective in other countries, ${ }^{5}$ and the same strategies may be effective in Japan.

Our study has some important limitations. While participating wards in this study represent a small sample of Japanese hospitals, these hospitals were located in various parts of Japan. Second, one hospital was unable to provide an objective assessment of urinary catheter appropriateness and was thus excluded from appropriateness calculations.

Despite its limitations, this national prospective study provides epidemiological information about the appropriate use of urinary catheters in Japanese non-ICU wards. Inappropriate use of urinary catheters is high. That a physician's order for placing the urinary catheter was present in only $10 \%$ of the patients evaluated is one key opportunity for improvement. A multimodal intervention may also help improve the appropriate use of urinary catheters in this setting, as such an approach has been successful elsewhere. ${ }^{1,10}$

\section{Declarations}

\section{Ethics approval and consent to participate}

All study methods were carried out in accordance with relevant guidelines and regulations (declaration of Helsinki). This is an observational study conducted within the usual practice. Therefore, the Institutional Review Board of Mito Kyodo General Hospital has waived the informed consent from the patients (17-21). This study protocol was also approved by Tokyo Metropolitan Tama Medical Center Institutional Review Board (29-126), Mitoyo General Hospital Institutional Review Board (17-CR01-036), Institutional Review Board of Shonan Kamakura General Hospital (TGE00924-024), Mimihara General Hospital Institutional Review Board (II17-4), and Senshunkai Hospital Institutional Review Board, respectively.

\section{Consent for publication}

Not applicable

\section{Availability of data and materials}

The datasets generated and analysed during the current study are available from the corresponding author on reasonable request.

\section{Competing interests}

The authors have no competing interests to declare.

\section{Funding}

None.

\section{Authors' contributions}

KK, SS, YT, and AK conceived the study concept and design. YT, YK, TF, SN, NK, NT collected data. KK, KF, and DR analyzed and interpreted the data. KK, YT, and AK coordinated and drafted the manuscript. KK, JM, SS, KF, DR, YT, and AK critically reviewed the manuscript and made some changes. All authors read and approved the manuscript. 


\section{Acknowledgments}

Kazuya Nagasaki contributed to implementing this study at Hospital A. Yuki Uenoyama and Shin Sugiyama contributed to the data acquisition at Hospital B and C, respectively.

\section{References}

1. Saint S, Greene MT, Krein SL, Rogers MAM, Ratz D, Fowler KE, Edson BS, Watson SR, Meyer-Lucas B, Masuga M, Faulkner K, Gould CV, Battles J, Fakih MG. A program to prevent catheter-associated urinary tract infection in acute care. N Engl J Med 2016; 374:2111-2119.

2. Magill SS, O'Leary E, Janelle SJ, Thompson DL, Dumyati G, Nadle J, et al. Changes in prevalence of health careassociated infections in U.S. hospitals. N Engl J Med. 2018;379(18):1732-44.

3. Saint S, Trautner BW, Fowler KE, Colozzi J, Ratz D, Lescinskas E, Hollingsworth JM, Krein SL. A multicenter study of patient-reported infectious and noninfectious complications associated with indwelling urethral catheters. JAMA Intern Med. 2018;178(8):1078-1085.

4. Meddings J, Rogers MA, Krein SL, Fakih MG, Olmsted RN, Saint S. Reducing unnecessary urinary catheter use and other strategies to prevent catheter-associated urinary tract infection: an integrative review. BMJ Qual Saf. 2014;23(4):277-89.

5. Meddings J, Saint S, Fowler KE, Gaies E, Hickner A, Krein SL, et al. The Ann Arbor Criteria for appropriate urinary catheter use in hospitalized medical patients: results obtained by using the RAND/UCLA Appropriateness Method. Ann Intern Med. 2015;162(9 Suppl):S1-34.

6. Kim B, Pai H, Choi WS, Kim Y, Kweon KT, Kim HA, et al. Current status of indwelling urinary catheter utilization and catheter-associated urinary tract infection throughout hospital wards in Korea: A multicenter prospective observational study. PLoS One. 2017;12(10):e0185369.

7. Kuriyama A, Takada T, Irie H, Sakuraya M, Katayama K, Kawakami D, et al. Prevalence and appropriateness of urinary catheters in Japanese intensive care units: results from a multicenter point prevalence study. Clin Infect Dis 2017;64(suppl_2):S127-S30.

8. Gould CV, Umscheid CA, Agarwal RK, Kuntz G, Pegues DA. Guideline for prevention of catheter-associated urinary tract infections 2009. Infect Control Hosp Epidemiol 2010;31:319-26.

9. Fowler KE, Krein SL, Ratz D, Zawol D, Saint S. Sustainability of a program to reduce unnecessary urethral catheter use at a Veterans Affairs hospital. Infect Control Hosp Epidemiol. 2021;Epub ahead of print.

\section{Supplementary Files}

This is a list of supplementary files associated with this preprint. Click to download.

- SupplementaryAppendixTable.docx 